Check for updates

Cite this: RSC Adv., 2017, 7, 42345

Received 23rd May 2017

Accepted 25th July 2017

DOI: $10.1039 / c 7 r a 05807 a$

rsc.li/rsc-advances

\title{
A nucleic acid logic gate system that distinguishes different sets of inputs from one miRNA collection with shared members $\uparrow$
}

\author{
You Zhang, ${ }^{a}$ Xiangjiang Zheng, ${ }^{b}$ Lian Xia, (D) ${ }^{a}$ Jinmao You*a and Rui Ren (D) *ab
}

A proof-of-principle logic system was established to process multi-input and multi-output logic relationships with the aim of identifying the tissue origins of cancer in light of their established relationships with miRNA distributions. The system detected two characteristic miRNA sets from a collection with two gates, each identifying a set of three input miRNAs, with miRNAs members shared among the two sets. The design of the logic system featured three stages: the initialization stage, the logic stage, and the signal stage. The initialization stage transduced each inputted miRNA into a messenger DNA in multiplied quantities. In the logic stage, the two logic gates were implemented; each picked up one set of inputted miRNA and produced the according RCA product. In the signal stage, the RCA products from the two gates were labelled with respective Raman tags, and the according SERS signal for each gate was produced. The logic system provided amplification for the miRNA inputs and thus was capable of processing miRNA with low abundance. The designed logic behavior was also verified to ensure that each gate only reacted to its specific input set, and the shared miRNA inputs could be processed by both gates. It was also verified that mismatched miRNA inputs did not interfere with the designed logic behaviors.

\section{Introduction}

Identifying the tissue origins of cancer is of great importance in the diagnosis and treatment of cancer, especially for cancers of unknown primary origin (CUP). The tissue origins of CUPs are difficult to identify because of metastasis, which resulted in cancer tumors occurring in places other than where they originated. ${ }^{1}$ Recently, relationships were established between miRNA distributions and the tissue origins of cancer. ${ }^{2,3}$ The expression distributions of miRNAs depend on the specific organism in which they occur. The miRNAs in cancer cells maintain the distribution patterns of the tissues or organisms where the cancer originated, regardless of metastasis. New approaches are thus expected for identifying the origins of cancer based on this relationship; this expectation has led to the following considerations and challenges.

In the established relationship, one cancer origin usually corresponds to more than one miRNA, and one miRNA is often related to more than one cancer origin. ${ }^{3}$ Thus, multi-input, multioutput systems are required. Such systems have been reported

${ }^{a}$ Key Laboratory of Life-Organic Analysis of Shandong Province, Qufu Normal University, Qufu 273165, P. R. China.E-mail: greeneese@163.com

${ }^{b}$ Shandong Province Key Laboratory of Detection Technology for Tumor Makers, School of Chemistry and Chemical Engineering, Linyi University, Linyi 276000, P. R. China $\dagger$ Electronic supplementary information (ESI) available. See DOI: 10.1039/c7ra05807a using techniques involving DNA logic gates and linear threshold gates; in these systems, a single gate usually accepts more than one input and accordingly produces one on/off output., Combination of multiple gates addressed the above requirement. For example, Qian et al. ${ }^{6,7}$ developed linear threshold complexes with the aim of providing small-scale artificial molecular models for the neural network algorithm. While this work was a prominent achievement at the fundamental level, it lacked efficiency and feasibility from the practical point of view. Notably, it required prolonged reaction time and high-concentration input. A system with practical improvements is thus needed.

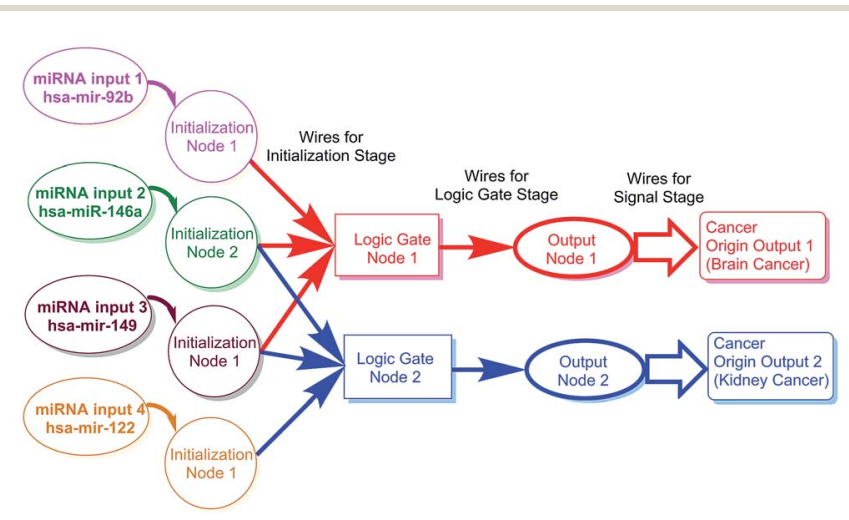

Scheme 1 Outline of the logic gate system in the study. The scheme also illustrates the relationship between miRNA distribution and cancer origin. 
The involvement of miRNA raises additional concerns. ${ }^{8}$ The abundance of miRNA in cells is usually very low, and miRNA is prone to degradation. Therefore, sensitive and degradationresistant techniques are required. Such techniques are not often encountered in previous studies on logic gates, such as Qian's report. ${ }^{6}$ In addition, miRNAs often occur in organisms in families with similar lengths and sequences (typically the let7 family) but with different functions. Thus, it is of vital importance for any miRNA assay to distinguish among such miRNAs. Various approaches have been developed to address these challenges, including transduction and/or amplification based on nucleic acid molecular machines ${ }^{9}$ and signal enhancement based on surface-enhanced Raman scattering (SERS). ${ }^{\mathbf{1 0}}$

\section{Principle and design}

The main purpose of the current study is to establish a proof-ofprinciple logic system that is capable of processing the

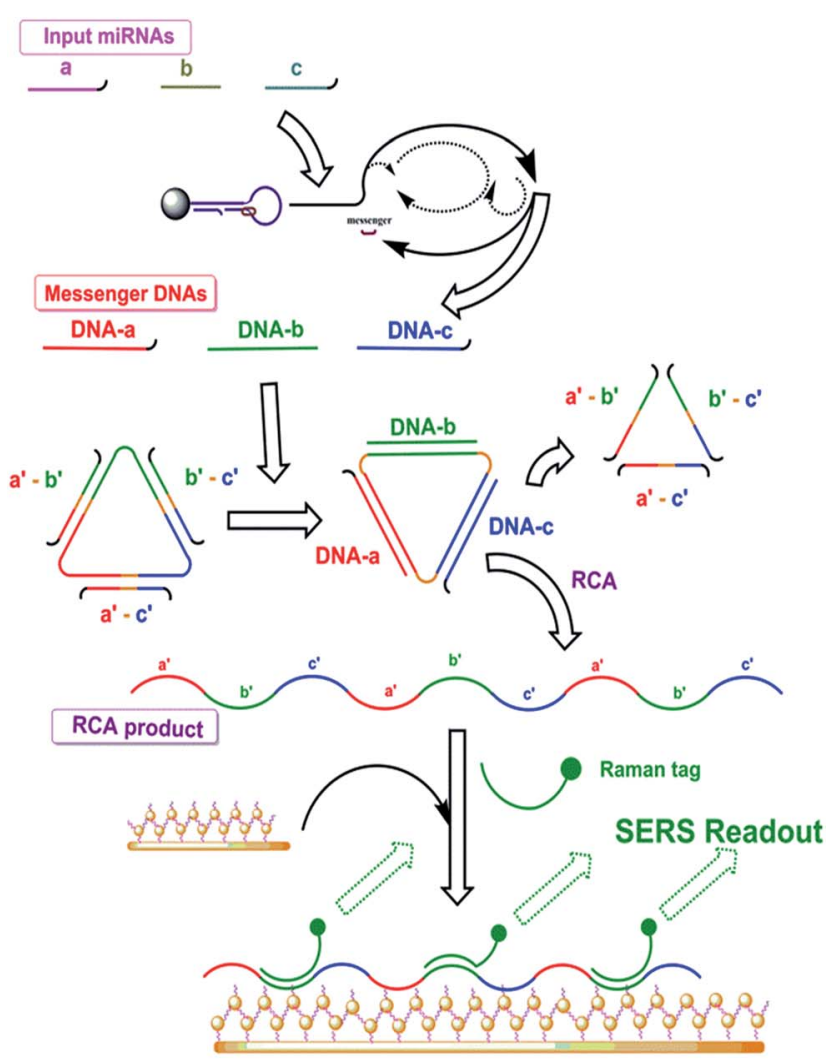

(a)

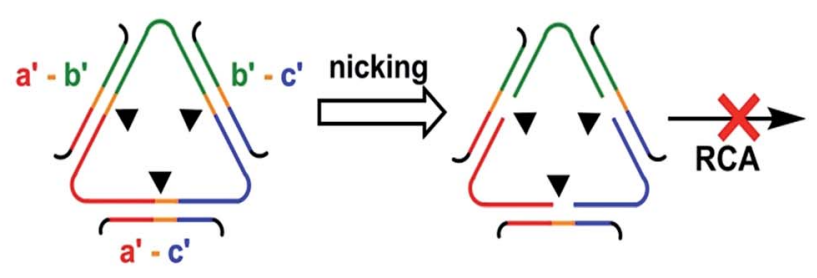

(b)

Scheme 2 Implementation of the logic system. The two most typical reaction paths are illustrated. (a) "On" path producing "on" output and (b) "off" path producing "off" output. aforementioned multi-input/multi-output logic relationship in the hope that it could be used in the detection of the characteristic miRNA sets from a collection. The overall aim is to identify the tissue origins of cancer in light of their established relationships with miRNA distributions.

The principle of this study featured a logic gate system that processed a collection of miRNA inputs. The collection contained four input miRNAs. These inputs fell into two sets, with each set corresponding to one origin according to the established relationship. Each set consisted of three members, and two of the miRNA inputs were shared among the two sets. The logic gate system consisted of two gates. Based on its own logic, each gate picked one characteristic set of miRNAs from the collection as its specific input and produced the corresponding on-off output, which served as the indicator of the corresponding cancer origin.

The shared miRNA members acted as the inputs of the two gates simultaneously. Considering this and the low abundances of miRNAs in organisms, at least these two members must be recycled or subjected to signal amplification. As miRNAs are prone to degradation, it is necessary to transduce the target into more stable forms, such as messenger DNA. Finally, signal production is also necessary to make the result of the logic gates and the amplifications readable.

The main purpose and principle of this study are outlined in scheme 1. The scheme also shows the relationship between miRNA distribution and cancer origin. The design consisted of three stages: the initialization stage, gate stage, and signal stage. For the convenience of description, each stage includes "nodes" and "wires." Each node consisted of all the materials needed for the stage. The wires, which usually linked nodes, consisted of the designed motions (e.g., reactions) needed for the stage to be carried out (Scheme 2).

\section{Implementation}

The implementation of the current study was shown in Scheme 2.

\section{Initialization stage}

The initialization stage was designed to recognize the input miRNAs and transduce them into messenger DNAs with multiplied quantities. The messenger DNAs, as the representatives for the input miRNAs, then served as the inputs for the following logic stage.

This stage was implemented with a protocol based on one of our previous reports. ${ }^{11}$ For the node, one probe and one primer were prepared for each input miRNA. Each probe consisted of a hairpin and a messenger DNA bound to the hairpin. For the wire, this stage started with the recognition of the target miRNA by the hairpin and resulted in the messenger DNA strand being released in multiplied quantities. The details of this stage are given in the ESI. $\dagger$

Logic stage. The logic stage involved the implementation of the logic gates. There were two gates, each of which picked up its corresponding set of messenger DNA inputs (representatives 
of the miRNAs from the initialization stage) and launched different reaction paths accordingly. This resulted in the on-off outputs: "on" output when the full set of inputs was present and "off" output otherwise. The outputs then served as switches for the following signal production and readout stage.

Each node centered on a single-stranded circular DNA template, and three "blocking" strands were also included. The structures of the probes were designed to support the operation of the wire. This stage was also assisted by nicking endonuclease, DNA polymerase, and other needed materials.

Because of the nature of the logic operations, each wire had at least two paths depending on the inputted messenger DNAs and the binding statuses, "off" and "on", for producing the on/ off outputs. Each path involved three steps: variation of the binding statuses, on/off nicking and on/off RCA. And each based on certain designs in the node.

Each path started from different binding statuses of the circular template with the messenger DNAs or with the binding strands. Various binding status might occur according to the input. The different binding statuses led to different behaviors regarding the nicking and RCA, thereby resulting in different outputs:

"Off" path: the template bound with the three "blocking strands" (as in the initial status). This typically occurred in the case that no messenger DNA (no input miRNA) was present. The binding of any blocking strand(s) on the circular template formed a functional nicking site, thus leading to the breaking of the template. Once the template was nicked at any point, it became unavailable for the RCA. Nicking and damaging of the template in massive quantities were expected to prominently lower the reaction level of RCA. In summary, from "off" binding status (where the circular template bound with three "blocking" strands), the three nicking sites were functional, caused "off" nicking, and led to the following "off" RCA (low content RCA product or none).

"On" path: typically, the template bound with the three messenger DNAs. This occurred when the entire set of characteristic miRNA inputs were present. Only the binding of the entire set of characteristic miRNA inputs (three) displaces and thus removed all the previously bound blocking strands, disabling the nicking sites, and thus the circular template would remain intact. The circular template remains intact, and RCA could carry on. In summary, from "on" binding status (i.e. when the whole set of messenger DNAs, as the substitutes of the pre-designed set of input miRNAs, were present and bound on the circular template), the blocking strands would be removed through hybridization displacements, causing "on" nicking, and leading to the following “on” RCA (producing RCA products).

The absence of one member in the set resulted in the inefficient removal of at least one blocking strand, leaving at least one nicking site functional. This resulted in "off" nicking and "off" RCA.

Signal stage. In the signal stage, SERS readouts were produced based on the RCA products from the logic stage. These products were labelled with Raman tags and then subjected to SERS signal enhancement. The readouts were then produced.

The nodes of this stage included the SERS matrix for the enhancement of the Raman signal and Raman tags for the production of the signal. The matrix was an assembly of the gold thin film and gold nanoparticles and was able to bind the RCA products through "capturing strand." Two Raman tags were also prepared, each for the signal production of one gate. Each tag was a DNA segment that could bind the corresponding RCA product through hybridization. Each tag was labelled with different Raman functional groups corresponding to different cancer origins.

For the wire: the RCA product was labelled with Raman tags, then captured on the enhancing matrix; and the entire complex was then quantified through SERS measurements. The Raman scattering signals were recorded on a Renishaw inVia Raman microscope.

The proof-of-principle miRNA collection contained hsa-mir92b (a), hsa-miR-146a (b), hsa-mir-149 (c) and hsa-mir-122a (d). According to the molecular biology report, they are related to two cancer origins in sets: the set of (a), (b) and (c) for brain cancer and the set of (b), (c) and (d) for kidney cancer. The collection of the four miRNAs was thus used as the input in this proof-of-principle study.

The outputs of the two gates associated in principle with the two cancers were labelled with two Raman tags, ROX (for brain cancer, characteristic Raman peak at $1499 \mathrm{~cm}^{-1}$ ) and malachite green (for kidney cancer, characteristic Raman peak at $1614 \mathrm{~cm}^{-1}$ ).

\section{Results and discussion}

\section{Amplification performance}

The amplification capacity (i.e., the ability of the system to sense miRNA inputs with low abundance) was first tested. The reaction system contained two major amplification mechanisms, exponential amplification in the initialization stage and RCA in the logic stage. These amplifications are both based on welldeveloped DNA molecular machine techniques.

The following investigations of amplification were all exemplified by one of the two gates, the one that produced readouts through ROX tag (since the performances of the two gates were assumed to be equal), with its input miRNA set served as target as a whole. The three miRNA members in the input set were always input with the same concentration, which was recorded as the target concentration for the following calibration curve and other quantitative experiments.

The optimal reaction conditions for amplification were determined to be a reaction time of $2 \mathrm{~h}$, a Phi29 DNA polymerase concentration of $0.60 \mathrm{IU} \mu \mathrm{L}^{-1}$, and a Nb.BbvCI (nicking endonuclease) concentration of $0.40 \mathrm{IU} \mu \mathrm{L}^{-1}$ (see Section S2.1 "Optimization of reaction conditions" and Fig. S4 in the ESI $\dagger$ ). Under the optimal conditions, the calibration curve was measured by plotting the normalized $\Delta I$, the SERS response at $1499 \mathrm{~cm}^{-1}$ (after subtracting the blank response) against the concentration of the target miRNA $\left(c_{\text {Target }}\right)$. A linear relationship was observed between the SERS response and the logarithm of target miRNA concentration across a concentration range of $10^{-16}$ to $10^{-14} \mathrm{M}$. In this range, the regression equation was $\Delta I$ $=4.78 \lg \left(c_{\text {Target }}\right)+76.86$, and the limit of the detection (LOD) was determined to be $7.6 \times 10^{-17} \mathrm{M}$ according to the $3 \sigma$ rule.

To explore the contributions of the two mechanisms of amplification, comparative amplification experiments were 
carried out. The SERS responses of the following modes were investigated across the respective concentration gradients:

(a) The complete reaction system was carried out;

(b) "RCA only" mode: The logic stage and signal stage were carried out using prepared messenger strands of known concentration as inputs instead of those produced in the initialization stage;

(c) "No amplification" mode: as a basepoint for the comparison of amplification, the SERS responses of ROX-tagged strands of known concentration were measured (i.e., in the case where no amplification was applied).

The results of the comparison experiments are shown in Fig. 1, while the SERS responses can be found in Fig. S6 and S7 in the ESI. $\uparrow$ The comparison of mode (b) and mode (c) indicated that RCA in the logic stage provided an amplification of about $10^{3}$ times. The comparison of mode (a) and mode (b) indicated that extra amplification was provided by the initialization stage: to reach the same SERS response, the target miRNA concentration at $10^{-14} \mathrm{M}$ grade was required in mode (a), while $10^{-11} \mathrm{M}$ was required in mode (b), thus at this response level, the initialization stage superposed the amplification of about $10^{3}$ times. The comparison of mode (a) and mode (c) showed that the total reaction system provided an amplification of about $10^{6}$ times. Thus, the system was verified to be capable of sensing miRNA inputs with low abundance.

The calibration curve for the "non-amplification" case (Fig. 1, curve C, details shown in the ESI $\dagger$ ) was defined by $I=$ $4.56 \lg (c)+47.01$ in the concentration range of $10^{-16}$ to $10^{-14} \mathrm{M}$. In contrast, the "RCA only" mode produced a calibration curve of $I=4.24 \lg (c)+56.46$.

\section{Logic behaviors}

The responses of the linear gates under different input configurations are the key proof for the system. Various cases were tested. The input sets of the two gates were tested (Fig. 2A and B,

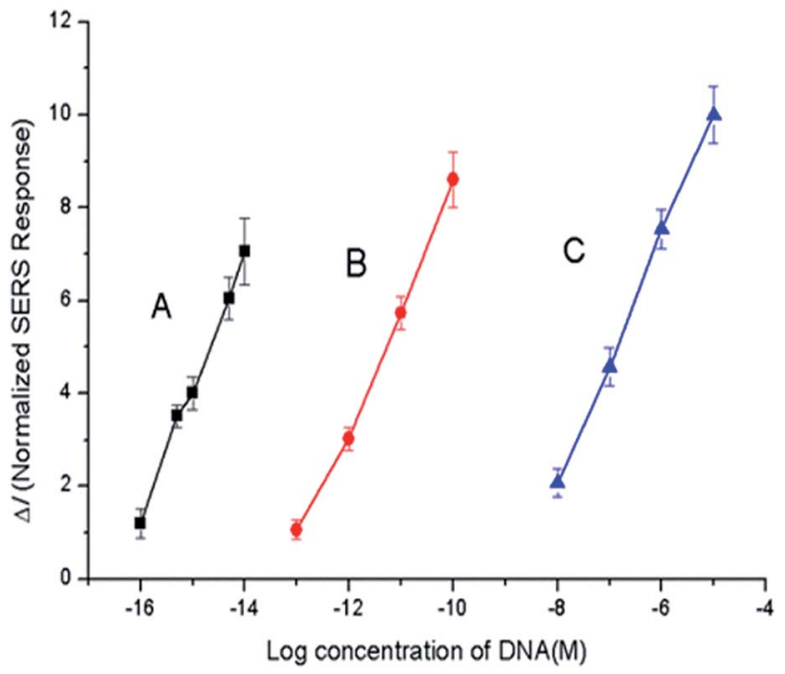

Fig. 1 Calibration curves of the amplification performances under different modes: (A) entire reaction system; (B) "RCA only" mode; and (C) "no amplification" mode. respectively). Only the gate that received its corresponding input set produced the positive SERS readout, while the other only showed the negative readout. The input of the entire collection containing all four miRNA members activated both gates, producing two positive readouts (Fig. 2C). Finally, as a control, the two shared miRNAs were input, and no positive readout was observed in either gate (Fig. 2D); although the two members can react in both gates, no input set was complete in this case.

\section{Interference of the logic behaviors}

One key requirement for the logic gate system is that a falsepositive readout should not be brought about by incorrect input miRNAs, especially in the case that miRNAs with similar sequences are present, which might occur in real organism due to the nature of the miRNAs. This was examined with modified selectivity experiments: one of the three miRNA members in an input set was replaced with mismatched miRNA, and the output of the logic system was examined.

The results (Fig. S12-S14 $\dagger$ ) confirmed the design: only the correct set of input miRNA produced positive output, while the mismatch of one or more members resulted in negative output.
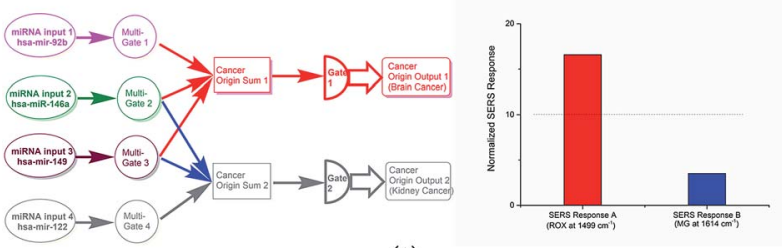

(A)
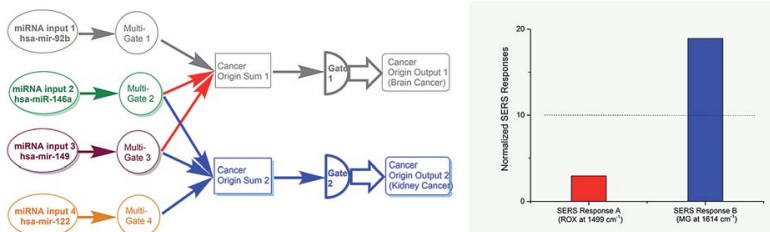

(B)
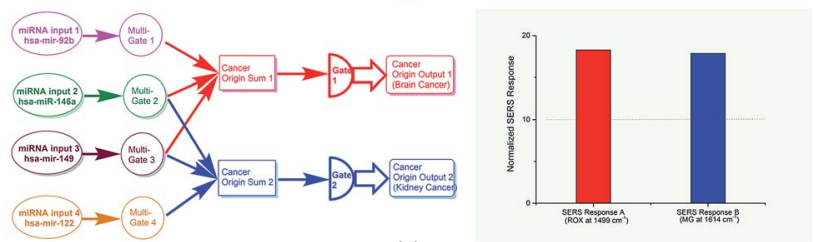

(C)
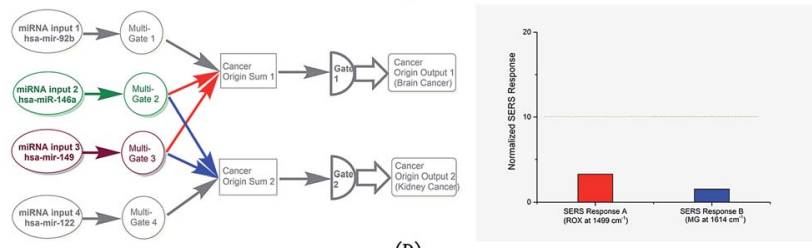

(D)

Fig. 2 The responses of the logic system. (A) Responses in the case that hsa-mir-92b, hsa-miR-146a and hsa-mir-149 co-exist. (B) Responses in the case that hsa-miR-146a, hsa-mir-149 and hsa-mir122 a co-exist. (C) Responses in the case that hsa-mir-92b, hsa-miR146a, hsa-mir-149 and hsa-mir-122a co-exist. (D) Responses in the case that hsa-miR-146a and hsa-mir-149 co-exist. 
Thus, the specificity of the approach was verified, and the specificity would not be affected under high amplification. The details of the experiments are shown in the ESI. $\dagger$

\section{Conclusions}

In summary, in this study, a proof-of-principle logic system was established to deal with the multi-input, multi-output logic relationship with the aim of identifying the tissue origins of cancer in light of their established relationships with miRNA distributions. The system did this by detecting two characteristic miRNA sets from a collection with two gates, each identifying a set of three input miRNAs with miRNA members shared among the two sets.

To fulfil its task, the system centered on logic judgement, which was implemented with a multi-input and multi-output logic gate complex. Signal amplification was also incorporated, providing the system with the practical capacity and efficiency to deal with miRNA collection. This system successfully addressed the multiple-input and multiple-output logic relationship between miRNA distribution and cancer origin. At the same time, other concerns related to the nature of miRNA (e.g., low concentration and instability) were also addressed.

\section{Acknowledgements}

This work was supported by the National Natural Science Foundation of China (No. 21205066).

\section{Notes and references}

1 (a) H. Blaszyk, A. Hartmann and J. Bjornsson, APMIS, 2003, 111, 1089-1094; (b) J. D. Hainsworth and F. A. Greco, N. Engl. J. Med., 1993, 329, 257-263; (c) J. M. Pimiento, D. Teso, A. Malkan, S. J. Dudrick and J. A. Palesty, Am. J. Surg., 2007, 194, 833-838; (d) P. H. S. Shaw, R. Adams, C. Jordan and T. D. L. Crosby, J. Clin. Oncol., 2007, 19, 8795; (e) G. R. Varadhachary, J. L. Abbruzzese and R. Lenzi, Cancer, 2004, 100, 1776-1785.

2 (a) S. Baskerville and D. P. Bartel, RNA, 2005, 11, 241-247; (b) J. Lu, G. Getz, E. A. Miska, E. A. Saavedra, J. Lamb, D. Peck, A. S. Cordero, B. L. Ebert, R. H. Mak, A. A. Ferrando, J. R. Downing, T. Jacks, H. R. Horvitz and T. R. Golub, Nature, 2005, 435, 834-838; (c) S. Volinia, G. A. Calin, C. G. Liu, et al., Proc. Natl. Acad. Sci. U. S. A., 2006, 103, 2257-2261.

3 N. Rosenfeld, R. Aharonov, E. Meiri, et al., Nat. Biotechnol., 2008, 26, 462-469.
4 S. Bi, Y. Yan, S. Hao and S. S. Zhang, Angew. Chem., Int. Ed., 2010, 49, 4438-4442.

5 R. Pei, E. Matamoros, M. Liu, D. Stefanovic and M. N. Stojanovic, Nat. Nanotechnol., 2010, 5, 773-777.

6 (a) L. Qian, E. Winfree and J. Bruck, Nature, 2011, 475, 368372; (b) L. Qian and E. Winfree, Science, 2011, 332, 11961201.

7 W. Li, Y. Yang, H. Yan and Y. Liu, Nano Lett., 2013, 13, 29802988.

8 (a) I. Lee, S. S. Ajay, H. Chen, A. Maruyama, N. Wang, M. G. McInnis and B. D. Athey, Nucleic Acids Res., 2008, 36, e27; (b) A. Valoczi, C. Hornyik, N. Varga, J. Burgyan, S. Kauppinen and Z. Havelda, Nucleic Acids Res., 2004, 32, e175.

9 (a) H. M. Chan, L. S. Chan, N. S. Wong and H. W. Li, Anal. Chem., 2010, 82, 6911-6918; (b) S. C. Chapin and P. S. Doyle, Anal. Chem., 2011, 83, 7179-7185; (c) Y. Cheng, X. Zhang, Z. Li, X. Jiao, Y. Wang and Y. Zhang, Angew. Chem., Int. Ed., 2009, 48, 3268-3272; (d) F. Degliangeli, P. Kshirsagar and V. J. Brunetti, J. Am. Chem. Soc., 2014, 136, 2264-2267; (e) R. J. Deng, L. H. Tang, Q. Q. Tian, Y. Wang, L. Lin and J. H. Li, Angew. Chem., Int. Ed., 2014, 53, 2389-2393; $(f)$ H. X. Jia, Z. P. Li, C. H. Liu and Y. Q. Cheng, Angew. Chem., Int. Ed., 2010, 49, 5498-5501; (g) S. Neubacher and C. Arenz, ChemBioChem, 2009, 10, 1289-1291; (h) L. Yang, C. Liu, W. Ren and Z. Li, ACS Appl. Mater. Interfaces, 2012, 4, 6450-6453.

10 (a) X. Kang, S. J. Ye, X. Y. Li, Y. Ma, C. Y. Zhang and B. Tang, Chem. Commun., 2016, 52, 5432-5435; (b) S. Lai, S. Wang, J. Luo, L. J. Lee, S. T. Yang and M. J. Madou, Anal. Chem., 2004, 76, 1832-1837; (c) M. Li, J. Zhang, S. Suri, L. J. Sooter, D. Ma and N. Wu, Anal. Chem., 2012, 84, 28372842; (d) X. Li, S. Ye and X. Luo, Chem. Commun., 2016, 52, 10269-10272; (e) Y. Li, C. Lei, Y. Zeng, X. Ji and S. Zhang, Chem. Commun., 2012, 48, 10892-10894; (f) Y. Li, X. Qi, C. Lei, Q. Yue and S. Zhang, Chem. Commun., 2014, 50, 9907-9909; $(g)$ G. Wang and R. J. Lipert, Anal. Chem., 2011, 83, 2554-2561; (h) S. J. Ye, Y. X. Yang, J. Xiao and S. S. Zhang, Chem. Commun., 2012, 48, 8535-8537; (i) S. J. Ye, Y. Y. Guo, J. Xiao and S. S. Zhang, Chem. Commun., 2013, 49, 3643-3645; (j) S. J. Ye, Y. Y. Wu, W. Zhang, N. Lia and B. Tang, Chem. Commun., 2014, 50, 9409-9412; (k) Z. Zhang, Y. Y. Wang, F. W. Zheng, R. Ren and S. S. Zhang, Chem. Commun., 2014, 51, 907-910.

11 R. Ren, Z. T. Yu, Y. M. Zou and S. S. Zhang, Chem.-Eur. J., 2012, 18, 14201-14209. 